\title{
HUGE THYROID SWELLING - HOW WE MANAGED THE SURGICAL DIFFICULTIES: A CASE REPORT
}

\author{
Faizanul Haque', Amit Kumar Ghosh², Bivas Adhikari3, Animesh Ghosh', Soumik Saha ${ }^{5}$
}

1 Post Graduate Trainee, Department of ENT \& Head and Neck Surgery, Calcutta National Medical College, Kolkata. ${ }^{2}$ Senior Resident, Department of ENT \& Head and Neck Surgery, Calcutta National Medical College, Kolkata. ${ }^{3}$ Associate Professor, Department of ENT \& Head and Neck Surgery, Calcutta National Medical College, Kolkata. ${ }^{4}$ Residential Medical Officer, Department of ENT \& Head and Neck Surgery, Calcutta National Medical College, Kolkata. ${ }^{5}$ Senior Resident, Department of ENT \& Head and Neck Surgery, Calcutta National Medical College, Kolkata.

\section{ABSTRACT}

Goiter means enlargement of thyroid gland. Endemic goitre due to iodine deficiency is a health problem that afflicts with varying degrees of severity, an estimated 40 million people throughout the Indian subcontinent.[1] The progressive nature of this disease occasionally results in severe tracheal compression and acute airway distress. Apart from goiters that are suspicious of cancer or possible cancer or inconclusive, surgery may be also recommended for those with benign biopsy results if the nodule is large, if it continues to increase in size or if it is causing symptoms (Pain, difficulty swallowing, etc.). Here, we report a case of large goitre with discussion on the difficulty faced during surgery, complications and how they were managed.

\section{KEYWORDS}

Huge Goitre; Tracheal Pressure; Thyroidectomy; Surgical difficulty; Complications.

HOW TO CITE THIS ARTICLE: Haque F, Ghosh AK, Adhikari B, et al. Huge thyroid swelling - how we managed the surgical difficulties: a case report. J. Evolution Med. Dent. Sci. 2016;5(17):885-889, DOI: 10.14260/jemds/2016/204

\section{INTRODUCTION}

The term "Goiter" simply refers to an abnormal enlargement of the thyroid gland. There are multiple causes of goiter, which may be benign or malignant. We continue to see a large number of patients with neglected goiters that cause airway compression. The progressive nature of this disease occasionally results in severe tracheal compression and acute airway distress.[2]

Thyroidectomy is an operation that involves the surgical removal of all or part of the thyroid gland. Surgeons often perform a thyroidectomy when a patient is having thyroid cancer or some other condition of the thyroid gland (Such as hyperthyroidism) or goiter. Other indications for surgery include cosmetic (Much enlarged thyroid) or symptomatic obstruction (Causing difficulties in swallowing or breathing).

Thyroidectomy, though a common surgical procedure, itself has several potential complications or sequelae including temporary or permanent change in voice, temporary or permanently low level of serum calcium, need for lifelong thyroid hormone replacement, bleeding, infection and the remote possibility of airway obstruction due to bilateral vocal cord paralysis. ${ }^{[3]}$ Beside the patient factors complications and difficulties experienced during the surgery depends on various factors such as size of the goiter, pathology of the swelling, experience of the surgeon, etc. Various studies have found that complications are less when the procedure is performed by an experienced surgeon. ${ }^{[4]}$

Here, we are reporting a case of large goiter which was managed by surgery with the discussion of complications and difficulties we faced during the surgery.

Financial or Other, Competing Interest: None.

Submission 25-07-2015, Peer Review 08-02-2016,

Acceptance 15-02-2016, Published 29-02-2016.

Corresponding Author:

Dr. Amit Kumar Ghosh,

148/c, Linton Street,

Kolkata-700014.

E-mail: amitkumarghosh1@gmail.com

DOI: $10.14260 /$ jemds/2016/204

\section{THE CASE}

A 42-year-old woman presented to ENT OPD with a huge thyroid swelling $(18 \times 14 \times 14 \mathrm{~cm})$ in front of her neck, which she had for the last 12 years. It had grown insidiously until a sudden spurt for the last two years. She was euthyroid and had mild respiratory distress at the time of presentation. Apart from the obvious disfigurement difficulty in respiration, her other problem was due to its enormous weight that caused a discomforting dragging sensation. It was huge single nodule with areas of firmness and cystic feeling. A CT scan of her neck revealed a well-defined heterogeneously enhancing space occupying lesion of $17 \times 12.2 \times 13.5 \mathrm{~cm}$ in size, arising from the left lobe and was extending up to the angle of the mandible and below anterior to the sternum. There was no obvious intrathoracic extension. The proximal trachea was slightly compressed and displaced to the right. (Figure: 1,2)

Endotracheal intubation although difficult, however, was successfully done with the help of fiber optic laryngoscope. Operation was done through a long transverse incision. The strap muscles were divided for better exposure. Difficulties faced to identify different vessels, recurrent laryngeal nerve and the parathyroid gland. The engorged large veins on the surface of the enlarged gland made it difficult to manipulate. However, extra care was taken during handling the gland to reduce intraoperative bleeding. All the vessels including the newly formed additional supply were ligated carefully. Recurrent laryngeal nerve in both sides identified and saved. Parathyroid glands saved in one side. (Figure: 3, 4). The trachea was palpated after the mass was removed and was found to be weakened in its cervical part.

The specimen weighed about 3.1kg. (Figure: 5) After taking out the mass we decided to perform a tracheostomy keeping in mind the fact that tracheomalacia may manifest after extubation as the trachea is weakened. A vertical incision of about 1.5 to $2 \mathrm{~cm}$ was made on the weakened part of the trachea. With the help of a tracheal dilator an $8 \mathrm{~mm}$ plastic cuffed tracheostomy tube was inserted into the trachea and the intubation tube was taken out; the cuff was then inflated and 
further anaesthesia was maintained through the tracheostomy tube. The histopathology revealed a colloid goitre. (Figure 6)

From the $7^{\text {th }}$ post-operative day, the patient developed serous collection at the incision site which was successfully managed by aspiration and pressure bandage. The tracheostomy was decannulated on $5^{\text {th }}$ postoperative day uneventfully.

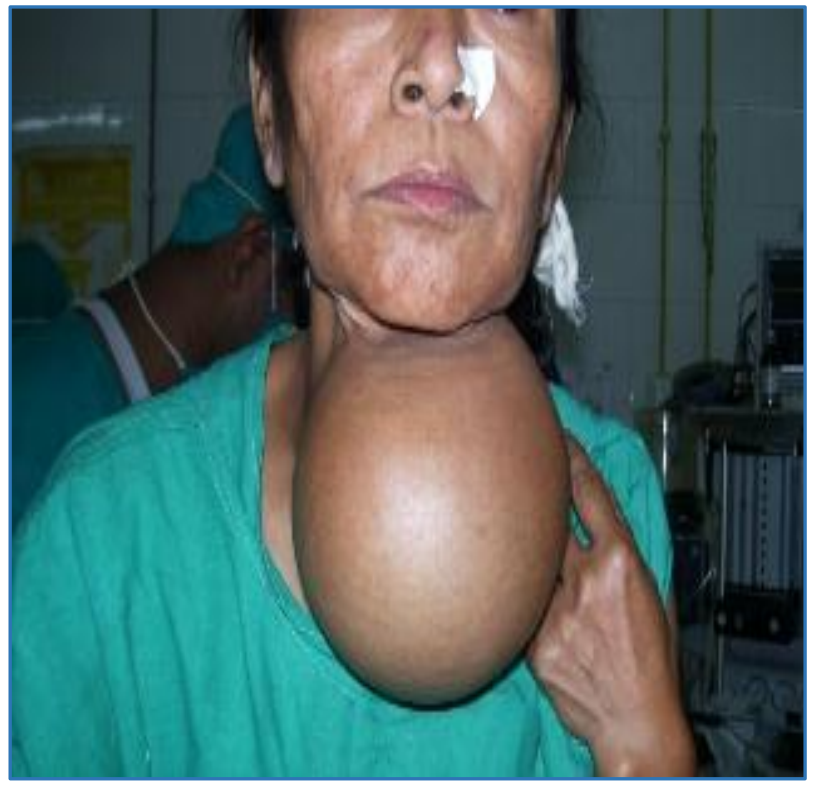

Fig. 1: Showing the Huge Goiter at Presentation

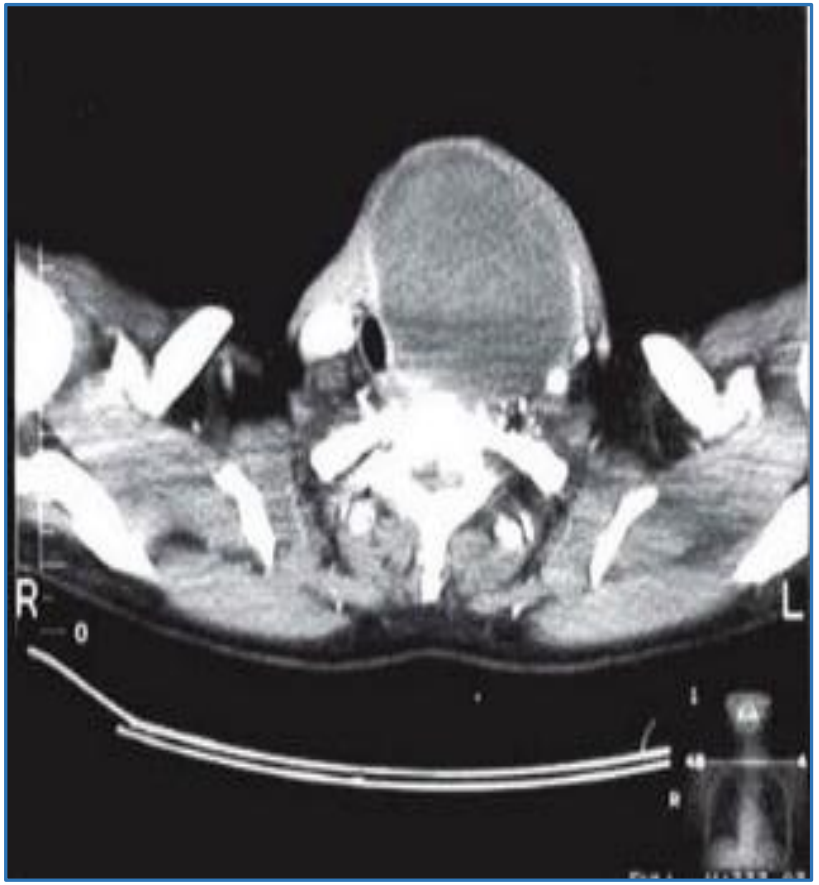

Fig. 2: CT Scan showing Deviation of the Trachea to the Right with Slight Compression

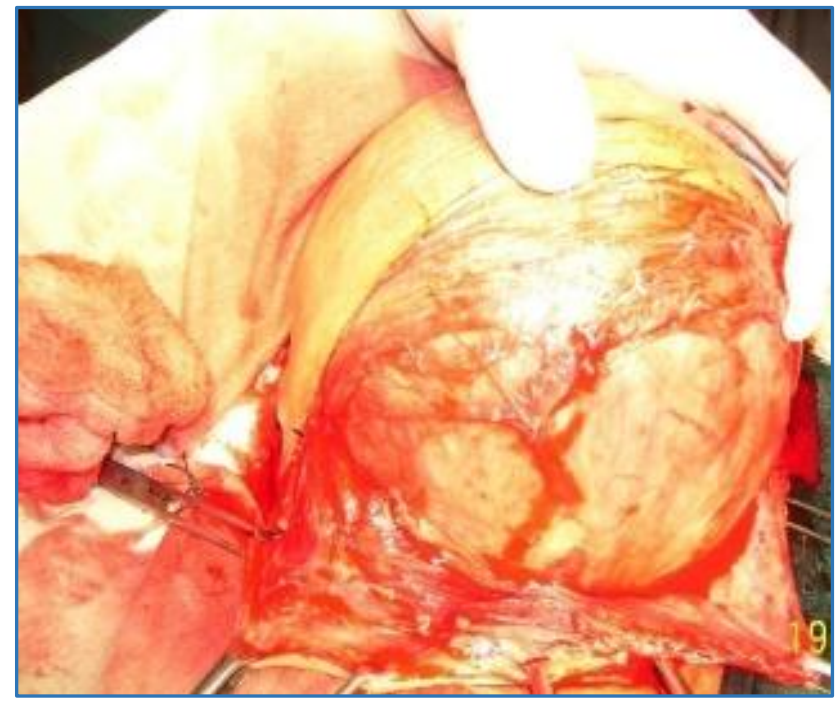

Fig. 3: Per-Operative Picture of the Same Patient

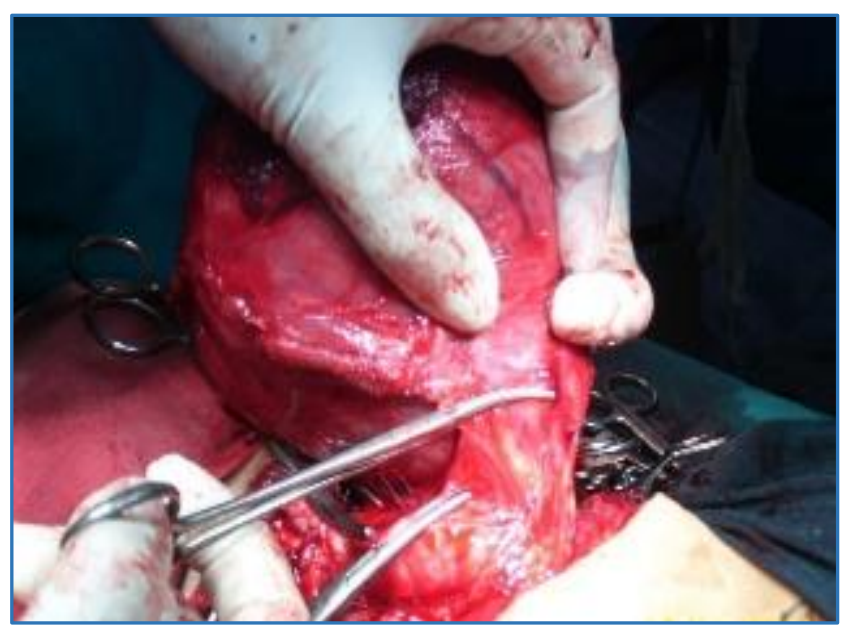

Fig. 4: Separating the Mass from its Last Attachment

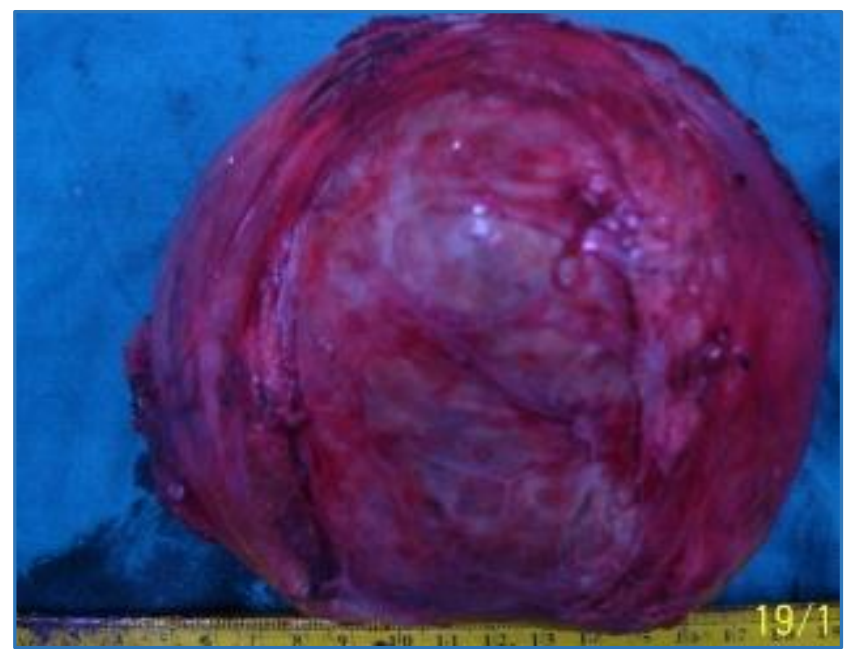

Fig. 5: Measurement of the Specimen with Scale 


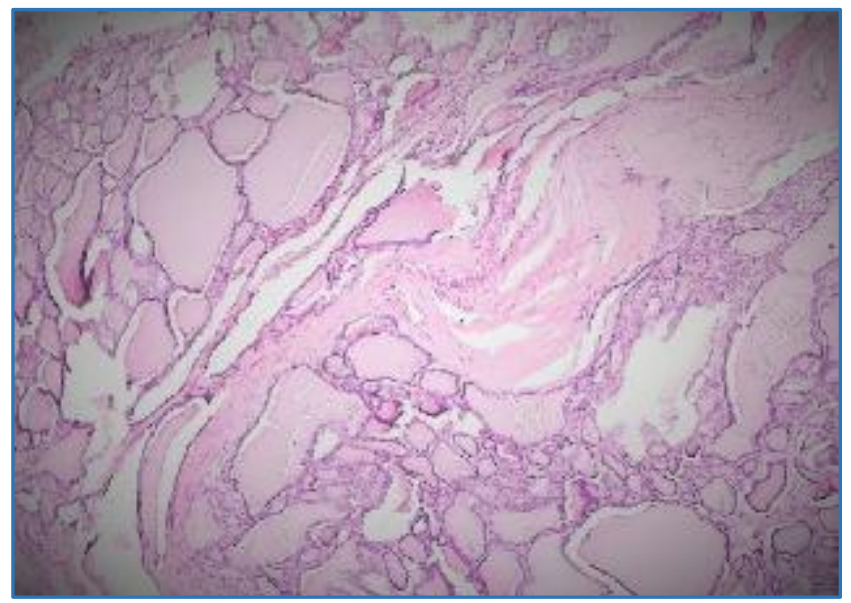

Fig. 6: Histology showing Colloid Goitre

\section{DISCUSSION}

The term "Goiter" simply refers to an abnormal enlargement of the thyroid gland. There are multiple causes of goiters and their presence does not necessarily indicate that the thyroid gland is malfunctioning on a hormonal level. These massive thyroid glands may be benign or malignant in nature. However, this particular case of huge thyroid swelling proved to be benign, which was a non-toxic colloid goitre.

As stated in UT-ORL newsletter and other studies, worldwide iodine deficiency is the leading cause of goiter formation. ${ }^{[5,6,7,8]}$ however, giant goiter is much less common. These patients have usually allowed the thyroid gland to grow to huge size with many years of neglect, but occasionally growth can be rapid. Rapid growth may be a harbinger of malignant pathology or may be due to hemorrhage into the gland. Here the patient suffered from goiter for twelve years, but it attained huge size within two years.

Surgical management of giant goiters is the same as routine thyroid goiters. Patient must be euthyroid before surgery. Large goiters cause multiple airway issues that may complicate surgery.[9] First, the trachea may be deviated and compressed by the large gland making intubation challenging. Generally, fiberoptic intubation is readily accomplished in these patients. Fiberoptic intubation can safely and promptly secure the airway and many studies have stated that it should be considered as an early option in difficult airway situation. ${ }^{[10,11,12]}$ Another problem with the airway is that, as the large thyroid typically covers the trachea along the entire cervical portion, these patients cannot be easily tracheotomized if the patient develops stridor; sometimes it may be impossible. However, unlike anaplastic carcinoma, very little has been published in the literature regarding performing an emergency pre-operative tracheostomy in these cases, as most of them could be managed by emergency fiberoptic endotracheal intubation with assisted ventilation.[13] Sometimes emergency thyroidectomy has also been done.[14] In this particular case though there was mild respiratory distress, no stridor was present and it could be easily managed by moist oxygen inhalation pre-operatively. Intubation was difficult and was done by fiberoptic laryngoscope before the surgery.

Long standing airway compression from goiters may also lead to tracheomalacia, which though rare is serious, even life threatening in the postoperative patient. Preoperative flexible fiberoptic laryngoscopy combined with tracheoscopy is gold standard for detection.[15] However, the symptoms and findings may not manifest until after the thyroid gland is removed. This is because fibrous attachments from the thyroid gland to the trachea may help to maintain airway patency during the breathing cycle. This external scaffolding is lost with thyroidectomy allowing the trachea to collapse. Till now, there is no generalized consensus on whether conventional tracheotomy is reasonable after the resection of a huge goitre. Conventional tracheotomy was not recommended except for the following cases: (1) Long-term compression of the trachea by huge goiter and destruction of more than 2 tracheal rings showed by CT scanning. (2) Obvious tracheal compression with narrowing of the lumen and there are difficulties in tracheal intubation and induction of anesthesia. (3) Preoperative recurrent laryngeal nerve palsy. (4) Weakening of trachea on palpation after removal of the thyroid or occurrence of tracheal collapse after the tumor resection. However, it should be remembered that intra-operatively the cartilage may seem rigid on palpation even after removal of the goiter; it is usually after thyroidectomy and extubation that these patients may develop stridor from dynamic collapse of the weakened trachea. Under these above mentioned circumstances, a preventive tracheotomy should be actively initiated and breath is assisted with ventilator, which better ensure patient safety. $[16,17,18]$

More recently, it has been argued that noninvasive positive pressure ventilation is effective and safe in the management of stridor and airway compromise following extubation in patients with post-thyroidectomy tracheamalacia.[19] However, this requires a well-equipped anaesthetic team, good instruments, critical monitoring and more importantly a good coordination between surgeons and anaesthetic team. In this case pre-operative tracheoscopy was not done because of limited facility, but after removal of the thyroid mass trachea was found to be weakened on palpation and a preventive tracheostomy was done to combat possible tracheomalacia.

There are multiple hemodynamic changes that occur with giant goiters. Usually, these occur when there is substernal extension. The gland may thus cause compression of the neck and upper mediastinal vessels causing venous outflow obstruction and superior vena cava syndrome. Another ramification of this venous congestion is that there can potentially be "Downhill varices" of the upper esophagus, which can lead to hematemesis. These engorged feeding vessels should be recognized intra-operatively and appropriately ligated to help prevent bleeding. There have even been numerous reports of cerebrovascular insufficiency and stroke from goiters either by direct compression of the carotid arteries or by "Thyrocervical steal" caused by increased thyroid blood flow. In these cases, thyroidectomy was curative of the ischemic events.

Incidences of intraoperative complications are higher in patients with large goiters when compared to patients with smaller thyroid glands.[20,21] These complications include higher rates of transient and permanent vocal cord paralyses as well as temporary and permanent hypocalcemia from parathyroid gland injury. Recurrent Laryngeal Nerve Palsy (RLNP) is an important and potentially catastrophic complication of thyroid surgery. Permanent RLNP occurs in 
$0.3-3 \%$ of cases with transient palsies in 5-8\%.[22,23] In our case no such complications developed in post-operative period. Risk of RLNP usually increases in revision thyroid surgery and surgery of thyroid malignancy. Risk of RLNP can be reduced by identifying the nerve by proper use of anatomical knowledge of the landmarks and use of nerve monitors. Neurologic injury from benign thyroid disease has been reported in cases of large benign goiter as well. These include phrenic nerve palsies, Horner's syndrome and recurrent laryngeal nerve palsies. No such complications detected in our case.

Another consideration that must be made in patients with giant goiters is poor nutritional status. The thyroid in these patients may either be compressing the esophagus directly via posterior extension or indirectly via cricotracheal compression posteriorly. Often, it is the resultant dysphagia which leads to poor nutrition. Poorly coordinated swallow may result and may persist after thyroidectomy and thus the surgeon should be mindful of the possibility of persistent dysphagia following surgery. Overall, the technical aspects of surgical management of large goiters are similar to those of the more common smaller goiter variety; however, difficulties as mentioned above may arise to operate on a large goiter. Surgeons who undertake these cases should be mindful of all of the potential complications and pitfalls.

\section{CONCLUSION}

Overall the principles of surgery, asepsis, hemostasis, careful dissection, restitution of function where possible and resuscitation should be followed. When surgeons performing thyroidectomy are knowledgeable about the thyroid gland, its function and its anatomy and are comfortable with its surgery, complications are low in number. Those that occur are largely correctible, thereby reducing the risk of litigation to a minimum among well informed patients. It is clear that to achieve all this, surgeons should be appropriately trained, follow best practice and maintain their level of commitment and good communication until the outcome is ensured. In this way the patient is not only satisfied, but appropriately relieved of the problem.

\section{REFERENCES}

1. Pandav CS, Kochupillai N. Endemic goitre in India: prevalence, etiology, attendant disabilities and control measures. March 1982, volume 49; pp 259-271.

2. Ashok R Shaha, MD, correspondence1; Carlos Burnett, MD1; Antonio Alfonso, MD1; et al. Goiters and airway problems: October 1989, volume 158, Issue 4, Pages 378380.

3. Sudarshan Babu, Lakshmi Shantharam KG. A study of complication of thyroidectomy. IJCRR 2013;5(17):95-101.

4. American College of Surgeons. Thyroidectomy complication rates are lower if surgeon performs 25 or more cases yearly. Medical Xpress; Oct 8, 2015 [accessed on 2016 Jan 4]. Available from: http://medicalxpress.com/news/2015-10-

thyroidectomy-complication-surgeon-cases-yearly.html

5. Department of Otorhinolaryngology-Head and Neck surgery, University of Texas Health Science Centre. Large benign goiters: special considerations for management. Newsletter [accessed on 2015 Dec 10]. Available from: https://med.uth.edu/orl/newsletter/large-benigngoiters-special-considerations-management/
6. Gregory A Ubom. The goitre-soil-water-diet relationship: case study in Plateau State, Nigeria. Article in science of the total environment. October 1991;107:1-11. DOI: 10.1016/0048-9697(91)90246-B.

7. Michael B Zimmermann. Research on iodine deficiency and goiter in the 19th and early 20th centuries. The Journal of Nutrition. November 2008; vol. 138(11):2060-2063.

8. Mark PJ Vanderpump. The epidemiology of thyroid disease. Br Med Bull. June 2011;99(1):39-51. doi: $10.1093 / \mathrm{bmb} / \mathrm{ldr} 030$.

9. Kirti Nath Saxena, Sudhir Kumar, Bharti Taneja, et al. Awake fibreoptic intubation in the sitting position in a patient with a huge goitre. Case reports in anaesthesiology, volume 2011 (2011), Article ID 352672, 3 pages.

10. Hariprasad M and Smurthwaite GJ. "Management of a known difficult airway in a morbidly obese patient with gross supraglottic oedema secondary to thyroid disease." British Journal of Anaesthesia, vol. 89, no. 6, pp. 927-930, 2002.

11. Chakera, Van Heerden PV and Van Der Schaaf A. "Elective awake intubation in a patient with massive multinodular goitre presenting for radioiodine treatment." Anaesthesia and Intensive Care, vol. 30, no. 2, pp. 236-239, 2002.

12. Stani Ajay Akkara, Ankit Singhania, Ajay George Akkara, et al. A study of flexible fiberoptic bronchoscopy aided tracheal intubation for patients undergoing elective surgery under general anaesthesia. Indian Journal of Otolaryngology and Head and Neck Surgery. April 2013; Volume 65, Issue 2, pp. 116-119.

13. Mellière D1, Saada F, Etienne G, et al. Goiter with severe respiratory compromise: evaluation and treatment. PubMed 1988 Mar;103(3):367-73.

14. Dalal S, Garg P, Jain A, et al. Multinodular goiter with acute dyspnea: is tracheostomy mandatory? The Internet Journal of Surgery 2006; Volume 11, Number 2.

15. Hartzell LD1, Richter GT, Glade RS, et al. Accuracy and safety of tracheoscopy for infants in a tertiary care clinic. Arch Otolaryngol Head Neck Surg. 2010 Jan;136(1):66-9. doi: 10.1001/archoto. 2009.204.

16. Barbuscia MA, Gorgone S, Di Pietro N, et al. Substernal goiter: pre, intra and postoperative problems. Chir Ital 2005;57:301. [PubMed]

17. ElBashier EM, Hassan Widtalla AB, ElMakki Ahmed M. Tracheostomy with thyroidectomy: indications, management and outcome: a prospective study. Int J Surg 2008;6:147-150. [PubMed]

18. Yabasin Iddrisu Baba1,2; Abass Adam3; Sam-Awortwi Wilfred4, et al. Perioperative airway management in the case of severe tracheal narrowing and deviation caused by multinodular goitre: case report. Journal of Anaesthesiology and Clinical Science.

19. Chi SY1, Wu SC, Hsieh KC, et al. Non-invasive positive pressure ventilation in the management of postthyroidectomy tracheomalacia. 2011 Sep;35(9):1977-83. doi: 10.1007/s00268-011-1178-4.

20. Ishtiaq Ahmed Chaudhary, Samiullah, Rehan Masood, et al. Recurrent laryngeal nerve injury: an experience with 310 thyroidectomies. J Ayub Med Coll Abbottabad 2007; 19(3):46.

http://www.ayubmed.edu.pk/JAMC/PAST/19-

3/12\%20Ishtiaq\%20Ahmed.pdf 
21. Bo Gao*, Wuguo Tian*, Yan Jiang, et al. Perioperative treatment of giant nodular goitre. Int J Med Sci 2012;9(9):778-785. doi:10.7150/ijms.5129.

22. Hayward NJ, Grodski S, Yeung M, et al. Recurrent laryngeal nerve injury in thyroid swelling: a review. ANZ J Surg 2013; Jan 83(1-2):15-21.
23. Riddell V (1970). Thyroidectomy, prevention of bilateral recurrent laryngeal nerve palsy. British Journal of Surgery 57, 1-11. 